\title{
Limb remote ischemic per-conditioning in combination with post-conditioning reduces brain damage and promotes neuroglobin expression in the rat brain after ischemic stroke
}

\author{
Changhong Ren ${ }^{\mathrm{a}, \mathrm{b}, \mathrm{f}, \mathrm{g}}$, Pengcheng Wang ${ }^{\mathrm{a}, \mathrm{c}}$, Brian Wang ${ }^{\mathrm{b}}$, Ning $\mathrm{Li}^{\mathrm{a}, \mathrm{f}, \mathrm{g}}$, Weiguang $\mathrm{Li}^{\mathrm{e}}$, \\ Chenggang Zhang ${ }^{\mathrm{e}}$, Kunlin Jin ${ }^{\mathrm{a}, \mathrm{b}}$ and Xunming Ji $\mathrm{j}^{\mathrm{a}, \mathrm{d}, \mathrm{f}, \mathrm{g}, *}$ \\ a Institute of Hypoxia Medicine, Xuanwu Hospital, Capital Medical University, Beijing, China \\ ${ }^{\mathrm{b}}$ Department of Pharmacology and Neuroscience, University of North Texas Health Science Center at Fort Worth, \\ Texas, USA \\ ${ }^{\mathrm{c}}$ Department of Neurosurgery, The Affiliated Hospital of Weifang Medical College, Weifang, Shandong, China \\ ${ }^{\mathrm{d}}$ Department of Neurosurgery, Xuanwu Hospital, Capital Medical University, Beijing, China \\ ${ }^{\mathrm{e}}$ Beijing Institute of Radiation Medicine, State Key Laboratory of Proteomics, Cognitive and Mental Health Research \\ Center, Beijing, China \\ ${ }^{\mathrm{f}}$ Center of Stroke, Beijing Institute for Brain Disorder, Beijing, China \\ ${ }^{\mathrm{g}}$ Beijing Key Laboratory of Hypoxia Conditioning Translational Medicine, Beijing, China
}

\begin{abstract}
.
Purpose: Limb remote ischemic per-conditioning or post-conditioning has been shown to be neuroprotective after cerebral ischemic stroke. However, the effect of combining remote per-conditioning with post-conditioning on ischemic/reperfusion injury as well as the underlying mechanisms are largely unexplored.

Methods: Here, adult male Sprague Dawley rats were subjected to middle cerebral artery occlusion (MCAO). The limb ischemic stimulus was immediately applied after onset of focal ischemia (per-conditioning), followed by repeated short episodes of remote ischemia $24 \mathrm{hr}$ after reperfusion (post-conditioning). The infarct volume, motor function, and the expression of neuroglobin $(\mathrm{Ngb})$ were measured at different durations after reperfusion.

Results: We found that a single episode of limb remote per-conditioning afforded short-term protection, but combining repeated remote post-conditioning during the 14 days after reperfusion significantly ameliorated cerebral ischemia/reperfusion injury. Interestingly, we also found that ischemic per- and post-conditioning significantly increased expression of Ngb, an oxygenbinding globin protein that has been demonstrated to be neuroprotective against stroke, at peri-infarct regions from day 1 to day 14 following ischemia/reperfusion.
\end{abstract}

\footnotetext{
${ }^{*}$ Corresponding author: Xunming Ji, MD, Department of Neurosurgery, Xuanwu Hospital, Capital Medical University, Chang Chun
}

Road 45, Beijing 100053, China. Tel.: +86 10 83198952; Fax: +86 10 63010085; E-mail: jixm@ccmu.edu.cn. 
Conclusion: Our results suggest that the conventional per-conditioning combined with post-conditioning may be used as a novel neuroprotective strategy against ischemia-reperfusion injury, and $\mathrm{Ngb}$ seems to be one of the important players in limb remote ischemia-mediated neuroprotection.

Keywords: Ischemia, stroke, remote conditioning, neuroglobin

\section{Introduction}

Ischemic stroke is the 4th leading cause of death in the United States and a leading cause of disability worldwide. The most recent major advancement for ischemic stroke treatment is the administration of tissue plasminogen activator (tPA) intravenously to dissolve clots. However, tPA appears to be effective only within the first 4.5 hours after the initial onset of symptoms (Moskowitz, et al., 2010), and only about $1-2 \%$ of stroke patients can benefit from this treatment. Although the understanding the basic molecular mechanisms underlying neuronal cell death have seen progress, subsequent clinical trials have been frustrating, and none of the neuroprotective agents have proven effective. Therefore, widely effective treatments for stroke remain elusive.

Recent studies have shown a new technique called remote ischemic conditioning, an intrinsic process whereby repeated short episodes of double-sided hind limb non-lethal ischemia/reperfusion is neuroprotective after global and focal cerebral ischemic stroke. Remote ischemic conditioning can be applied before ischemia (pre-conditioning; PreC), during ischemia and before reperfusion (per-conditioning; PerC), or during reperfusion (post-conditioning; PostC) (Hausenloy and Yellon, 2011). All three remote ischemic conditioning strategies have similar therapeutic potential, and could trigger innate mechanisms to protect against brain injury after ischemic stroke (Hoda et al., 2012; Zhao, 2009). However, ischemic PreC has limited clinical application due to the unpredictability of the ischemic event. Remote limb ischemic PerC and PostC are preferred for the treatment of acute ischemic stroke in the clinical setting as they can be applied during or after the ischemic period (Gidday, 2006; Veighey and Macallister, 2012; Yu, et al., 2012). Our group and Hoda et al. reported that remote limb ischemic per-conditioning ameliorated neuronal injury after focal ischemic stroke (Hoda, et al., 2014, 2012; Ren, et al., 2012), and daily remote limb ischemic postconditioning prevented recurrent stroke in intracranial arterial stenosis patients (Meng, et al., 2012).
Interestingly, Wei et al. reported that remote PerC in combination with daily remote PostC reduced adverse left ventricular remodeling and improved survival in a rat model of myocardial infarction, and that ischemic conditioning treatment was more effective than a single episode of remote pre-conditioning (Wei, et al., 2011). This suggests that ischemia PerC in combination with post-conditioning could protect against brain injury after ischemic stroke. In contrast, another recent study showed that the combination of remote perconditioning and post-conditioning failed to reduce infarct size in a rat model of coronary artery occlusion when compared to the untreated ischemia-reperfusion rat (Sachdeva, et al., 2014). Thus, whether the combined effect of remote PerC in combination with PostC on cerebral ischemic injury remains unknown.

Yet, PerC and PostC protect against ischemiareperfusion injury, mechanisms of how blood pressure cuff inflation on the leg lead to neuroprotection during ischemic stroke remains largely unexplored. Studies have shown that ischemia/hypoxia pre-conditioning could enhance the expression of hypoxia induced factor-1 (HIF-1) and vascular endothelial growth factor (VEGF) (Gidday, 2006). Consequently, HIF-1 and VEGF could increase the expression of neuroglobin $(\mathrm{Ngb})$, a newly discovered member of the hemebinding globin superfamily (Burmester, et al., 2000; Haines, et al., 2012; Jin, et al., 2012; Yu, et al., 2012). In the brain, the functions of $\mathrm{Ngb}$ are sensing oxygen, storage and/or transportation of oxygen, and ROS scavenging (Hua, et al., 2010; Yu, et al., 2012). Our previous studies have shown that $\mathrm{Ngb}$ is an endogenous critical neuroprotective molecule against ischemic/hypoxic insults (Jin, et al., 2010; Sun, et al., 2001; Sun, et al., 2003). Enhanced Ngb gene expression reduced infarct size and free radical formation following ischemia in murine experimental models (Khan, et al., 2006; Li, et al., 2010; Sun, et al., 2003; Wang, et al., 2008). In addition, overexpression of Ngb in cultured cells can protect neurons against hypoxic insults (Jin, et al., 2008; Liu, et al., 2009). Given the central role of $\mathrm{Ngb}$ in neuroprotection after ischemic stroke, we speculated that Ngb might be involved in remote 
per- and/or post-conditioning-mediated neuroprotection.

In this study, we investigated that combined effect of remote ischemic PerC and PostC on neurological deficits and on $\mathrm{Ngb}$ expression the ischemic brain after focal ischemia.

\section{Materials and methods}

\subsection{Animals}

All animal experiments were approved by the Animal Care and Use Committee of Xuanwu Hospital, Capital Medical University and conducted according to guidelines by the National Institutes of Health. Adult male Sprague-Dawley (280 to $320 \mathrm{~g}$ ) rats were purchased from Vital River, a Charles River company. Animals were housed in groups of 4 in the same animal care facility throughout the duration of the study, and were maintained on a 12-hour light/dark cycle with unlimited access to food and water. All efforts were made to minimize any suffering and to reduce the total number of animals used.

\subsection{Focal cerebral ischemia}

Male Sprague-Dawley rats (280 to $320 \mathrm{~g}$ ) were anesthetized and MCAO was induced by intraluminal occlusion for 90 min using a nylon monofilament suture as described previous (Zwagerman, et al.). In brief, the right common carotid artery and the right external carotid artery (ECA) were exposed. The ECA was then dissected distally, ligated, and coagulated. The MCA was occluded using a heparinized intraluminal filament $(0.28 \mathrm{~mm}$, rounded tip). After $90 \mathrm{~min}$, the suture was withdrawn. During the operation, rectal temperature was maintained at $37 \pm 0.5^{\circ} \mathrm{C}$ with a thermostat-controlled heating blanket. Physiological variables such as blood pressure, and blood gases ( $\mathrm{pH}, \mathrm{pO} 2$ and $\mathrm{pCO} 2$ ) were monitored before and after surgical procedures. Sham-operated mice underwent the identical surgery except that the MCA was not occluded.

\subsection{Remote ischemic per- and post-conditioning}

Rats were assigned to four groups including sham group, ischemic control group, ischemic PerC only group and ischemic per- and PostC group.

PerC was initiated immediately following the onset of focal ischemia by bilaterally occluding blood flow to the hind limbs (Zhang, et al., 2014). Hind limb occlusion was accomplished by tightening a tourniquet $(8 \mathrm{~mm})$ around the upper thigh for 3 cycles, with each occlusion or release phase lasting 10 minutes.

PostC was applied at 1 day after reperfusion and then repeated every day thereafter up to 14 days. Rats were anesthetized with sodium pentobarbital (30 mg/kg intraperitoneally) before PostC, as previously described (Wei, et al., 2011). The sham and ischemic control groups received the same dosage of sodium pentobarbital.

\subsection{Neurobehavioral test}

Neurological deficit was determined through the neurobehavioral scoring system developed by Belayev et al. (Belayev, et al., 1996) with modification. The scoring system was graded on a scale of 0 to 12 (minimal score, 0 ; maximal score, 12). The tests included (1) postural reflex test, to examine upper body posture, and (2) the forelimb placing test, to examine sensorimotor integration. Eight rats were used for each group.

The ladder rung walking test was also used to assess deficits in coordination and integration of motor movement and was performed as previously described (Sun, et al., 2012b). The number of errors and steps of the affected left forelimb in each trail was counted. Two trials were averaged to give a mean foot fault score, and testing was performed at 1, 7, and 14 days after reperfusion by an investigator who was blinded to the experimental groups. Higher scores indicate worse deficits. Eight rats were used for each group.

The elevated body swing test (EBST) was used to test asymmetric motor behavior (Borlongan and Sanberg, 1995). The rats were held at the base of the tail and raised $15 \mathrm{~cm}$ above the testing surface. The initial direction of swing is defined as the turning of the upper body by $>10$ degrees to either side, and was recorded in 30 trials per rat. The number of turns to each direction (left or right) was recorded for each rat. The total number of swings made to the left was divided by 30 ( $n$ number of trials) to get a percentage of left-biased swings. Eight rats were used for each group.

The observer was blinded to the experimental conditions while performing the neurobehavioral tests.

\subsection{Histology}

Each group rats ( $N=5$ per group) were anesthetized and decapitated at day 7 and 14 after MCAO. Brains 
were removed and $20-\mu \mathrm{m}$ coronal sections were prepared and stained with cresyl violet. Infarct area (7 days after reperfusion) or area of brain injury (14 days after reperfusion), contralateral hemisphere area, and total cross-sectional brain area were measured by a blinded observer using the ImageJ software (National Institutes of Health, Bethesda, MD), and areas were multiplied by the distance between sections to obtain the respective volumes. Infarct volume or area of brain injury was calculated as a percentage of the volume of the contralateral hemisphere, as described previously (Sun, et al., 2012a).

\subsection{TUNEL assay}

Each group rats ( $N=5$ per group) were anesthetized and decapitated at day 3 after MCAO. Brains were removed and 5- $\mu \mathrm{m}$ coronal paraffin-embedded sections were prepared. In situ detection of DNA fragmentation in brain tissues after ischemia was performed using Terminal deoxynucleotidyl transferase dUTP nick end labeling (TUNEL) detection kits (Roche. German) according to the manufacturer's protocol. Green-staining cells in the peri-infarct region were counted as apoptotic cells. The quantitative analysis was expressed using the average of four brain slides, and each slide included four random fields.

\subsection{Measurement of intracellular ROS}

Production of ROS was assayed using the ROS Fluorescent Determination Kit (GenMed Scientifics Inc., Arlington, MA, USA) (Ren, et al., 2013). At day 3 after MCAO, samples were prepared according to manufacturer's instruction. In brief, peri-infarct brain regions were lysed in Solution C. The obtained supernatant was pipetted into the wells of a 96-flat-bottom well microtiter plate containing DCF-DA (Solution C). The samples were incubated for $20 \mathrm{~min}$ at $37^{\circ} \mathrm{C}$ in dark, and then read in a Thermo Scientific Varioskan Flash with excitation at $490 \mathrm{~nm}$ and emission at $520 \mathrm{~nm}$ (Thermo Labsystem, Helsinki, Finland). Total protein concentration was measured with a Bradford Kit (GenMed Scientifics Inc., Arlington, MA, USA). ROS were calculated as absorption value per mg of protein and expressed as the relative value to the sham animals. Four rats in each group were used during the analysis.

\subsection{Western blot analysis}

Western blot analysis was used to assess $\mathrm{Ngb}$ expression. Protein was isolated from the rat periinfarct region at 1,7 , and 14 days after reperfusion. Protein $(20 \mu \mathrm{g})$ was electrophoresed on $12.5 \%$ SDSpolyacrylamide gels, and then transferred to a polyvinylidene difluoride membrane (Millipore Corporation, USA). The membrane was probed with rabbit polyclonal anti-Ngb antibody as described in our previous report (made in-house and validated) (Sun, et al., 2001). The specific reaction was visualized through the use of a chemiluminescent substrate (GE Healthcare, UK). $\beta$-actin was used to verify equal loading. The optical density of protein was measured using ImagePro Plus software 5.0 (Rockville, MD, USA) according to the manufacturer's instruction.

\subsection{Immunohistochemical analysis}

The rats $(N=5$ per group) were anesthetized with $10 \%$ chloral hydrate and were subsequently sacrificed by transcardiac perfusion with physiological saline followed by $4 \%$ paraformaldehyde dissolved in PBS $(\mathrm{pH}$ 7.4). The frozen sections ( $15 \mu \mathrm{m}$ thick) were incubated with rabbit polyclonal anti-NGB antibody (1:200 dilution) in PBS containing $0.03 \%$ Triton $\mathrm{X}-100$ at $4{ }^{\circ} \mathrm{C}$ overnight (Sun, et al., 2001). After washing, the sections were subsequently incubated with Alexa Fluor 488 donkey anti-rabbit IgG for $1 \mathrm{~h}$ at room temperature (Molecular Probes, 1:500). Slides were mounted using ProLong Gold anti-fade reagent with DAPI (Molecular Probes). The quantitative analysis was expressed using the average of four brain slides. Ngb-positive signal within the ipsilateral cortex peri-infarct region were examined and quantified using Image-Pro Plus software 5.0 in a blinded manner at 1, 7, and 14 days after reperfusion.

\subsection{Statistical analysis}

Data were expressed as mean and standard deviation (Mean \pm SD) and statistical tests were performed with SPSS for Windows, version 19.0 (SPSS Inc.). For comparison between two groups, Student's $t$ test was used. For behavioral tests, Kruskal-Wallis ANOVA was used, followed by the non-parametric MannWhitney test for comparison between two groups. In all cases, $p<0.05$ was the criterion for significance. 


\section{Results}

To determine whether limb remote ischemic PerC and PostC prevented ischemic injury after focal ischemia (Fig. 1B), infarct size or area of brain injury was measured at 7 and 14 days after reperfusion, respectively.

At day 7 after reperfusion, infarct volume was significantly reduced in the PerC and PerC+PostC groups compared with control group $(P<0.01)$. There was no significant difference between the PerC and PerC + PostC groups $(P>0.05)$ (Fig. 1B and 1C). These results suggest that PerC or the combination of PerC with PostC could influence acute histological outcome after MCAO.

At day 14 after reperfusion, the brain injury volume was significantly reduced in PerC + PostC group compared with control and PerC groups $(P<0.01)$ (Figs. 1D and 1E). However, there was no significant difference between the control and PerC groups. These results suggest that only PerC+PostC treatment could influence long-term histological outcome after MCAO.

To further analyze the neuroprotective effect of lime remote ischemic PerC and PostC, DNA fragmentation in brain tissues after ischemia was performed using TUNEL assay. The number of TUNEL-positive cells in the per-infarct region in the control group were significantly increased compared to the sham group $(P<0.01)$ (Fig. 2A and B). However, PerC and PostC treatment significantly decreased the number of TUNEL-positive cells compared with the control group $(P<0.01)$ (Fig. 2A and B). There was no significant difference between the PerC and PerC+PostC groups $(P>0.05)$.

Subsequently, we measured ROS level in these rats groups. As shown in Fig. 2C, the ROS level of control group was significantly higher than sham rats $(P<0.05)$. The ROS level was significantly repressed in the PerC group and Per+PostC group compared with control group or with sham group $(P<0.05)$. There was no significant difference between the PerC and PerC+PostC groups $(P>0.05)$.

Next, we asked whether combining PerC and PostC treatment would affect the neurological functional outcome. As shown in Fig. 3A, neurological deficits, including body posture and sensorimotor integration, were significantly improved at 7 and 14 days after reperfusion in the PerC+PostC group, compared with control group. Similarly, motor deficits were also improved at 7 and 14 days after PerC+PostC treat- ment based on the ladder walking test (Fig. 3B). The EBST also showed that PerC+PostC treatment improved asymmetric motor behavior at 7 and 14 days after reperfusion (Fig. 3C). However, the PerC only treatment had no effect on long-term neurological outcome after MCAO (Fig. 3).

Then, we explored the mechanisms underlying combinatorial therapy-mediated (PerC+PostC) neuroprotection after ischemic stroke. By Western blot analysis, we found that Ngb expression was significantly increased in the rat brain after focal ischemia, consistent with a previous finding (Jin, et al., 2010). The expression of $\mathrm{Ngb}$ was increased after PerC treatment at day 1 after reperfusion in peri-infarct region. Interestingly, $\mathrm{Ngb}$ expression dramatically increased after combination treatment of PerC+PostC at days 7 and 14, compared with sham and ischemic control groups (Fig. 4). However, the expression of Ngb in PerC group reduced to the baseline level at day 7 and day 14, suggesting that PerC + PostC treatment but not PerC-only treatment influences Ngb expression. Immunohistochemical analysis confirmed that $\mathrm{Ngb}$ expression was increased in the Perc+PostC group, compared with sham and control groups (Fig. 5).

\section{Discussion}

In this study, we found that a single episode of limb remote per-conditioning afforded short-term protection, but combining repeated remote post-conditioning during the 14 days after reperfusion significantly ameliorated cerebral ischemia/reperfusion injury through increased $\mathrm{Ngb}$ expression in the ischemic peri-infarct region, suggesting the benefit of combination therapy and $\mathrm{Ngb}$ involvement in PerC+PostC-mediated neuroprotection after focal ischemia.

Although pre-conditioning remains one of the most powerful maneuvers to protect brain damage, it is not feasible in the clinical setting to pretreat patients prior to acute ischemic stroke. Therefore, remote PerC and PostC are more clinically relevant therapies. A clinical study showed that remote PerC applied to the arm; by means of blood pressure cuff inflation during ambulance transport (i.e. during ischemia) increased the mean and median salvage indexes (Botker, et al., 2010). In addition, our recent study demonstrated that repeated daily remote PerC by limb ischemia prevents recurrent stroke in intracranial arterial stenosis patients (Meng, et al., 2012). Wei et al. reported that a single 


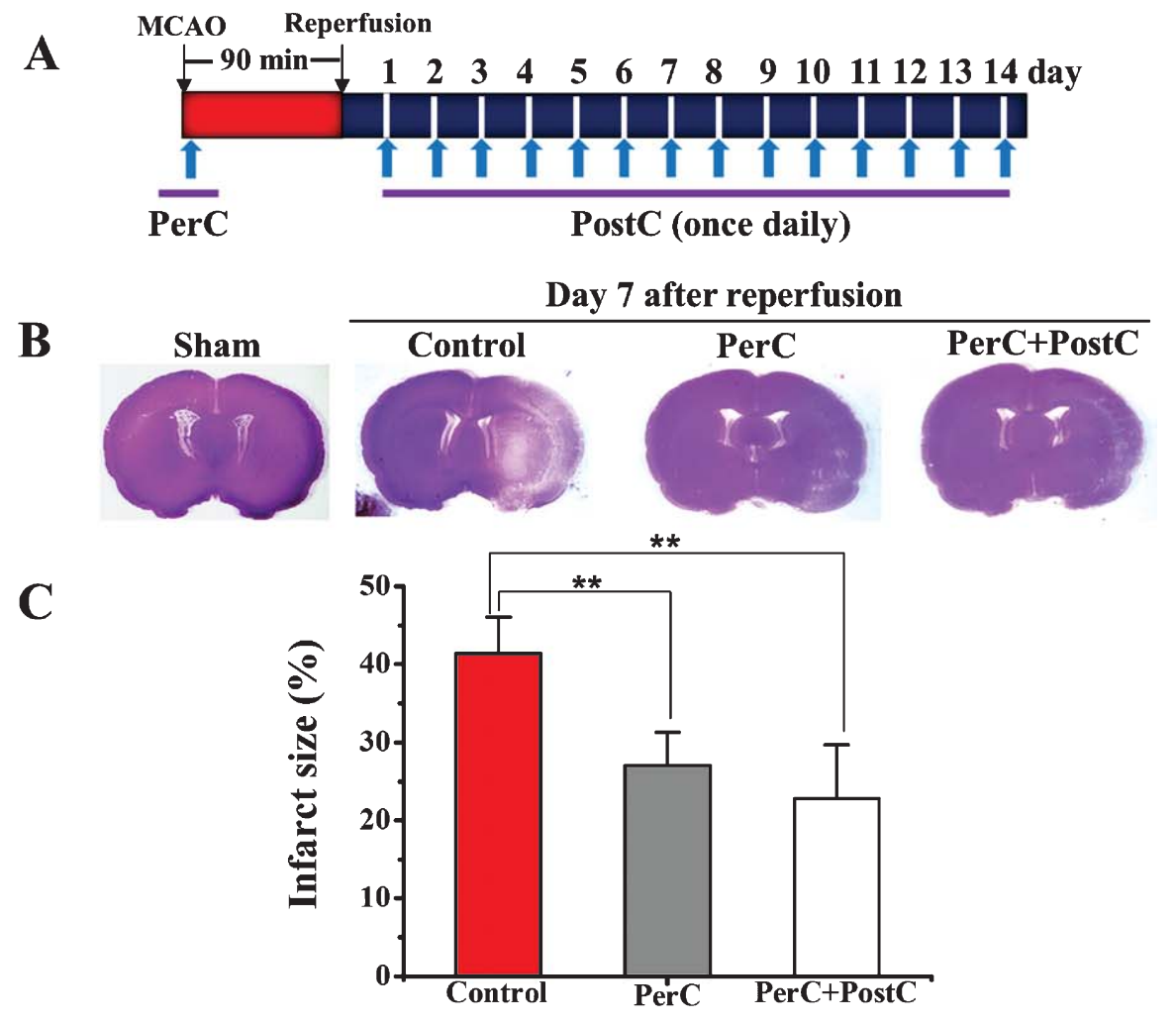

D

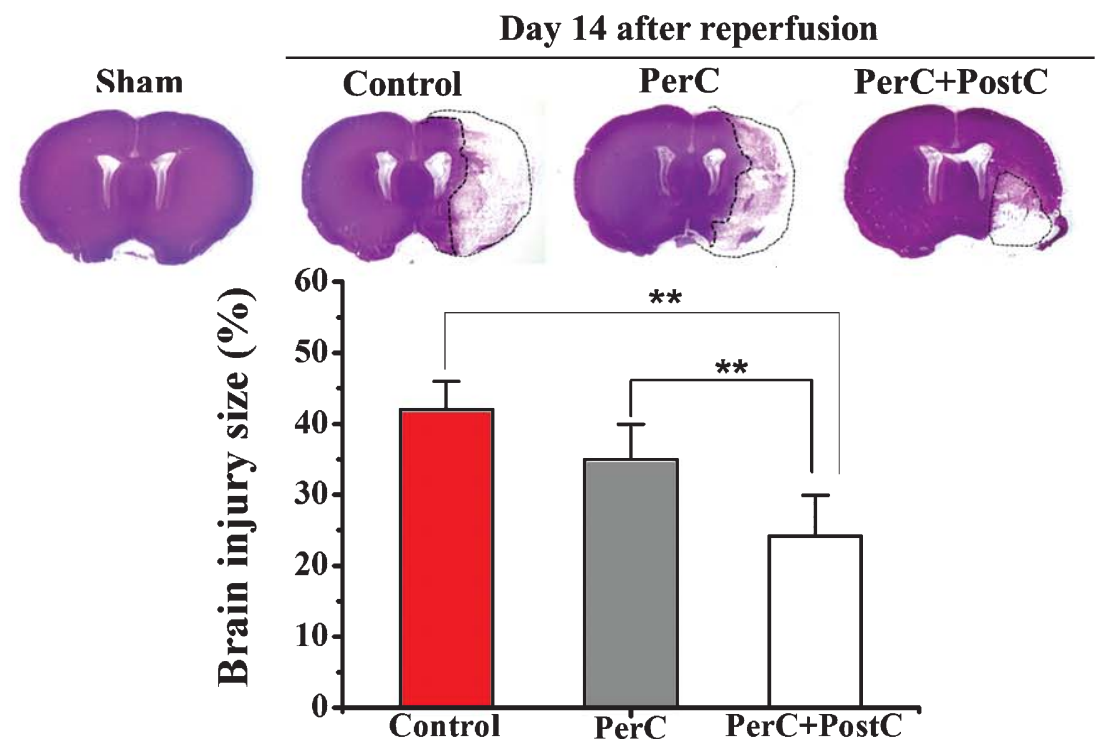

Fig. 1. Effect of per-conditioning and post-conditioning on infarct volume and area of brain injury. A, Schematic diagram showing limb remote ischemic conditioning, which was induced during ischemia (PerC) and once daily after reperfusion (PostC). B, Representative cresyl violet staining for infarct area measured at 7 days after reperfusion. C, Quantification of average infarct volume shown in B. ${ }^{* *} P<0.01$. Error bars indicate SD. $N=5$ per group. D, Representative cresyl violet staining for brain injury measured at 14 days after reperfusion. E, Quantification of average area of brain injury shown in D. ${ }^{* *} P<0.01$. Error bars indicate SD. $N=5$ per group. Control: ischemic control group. PerC: per-conditioning group. PerC+PostC: per-conditioning combined with post-conditioning group. 
A

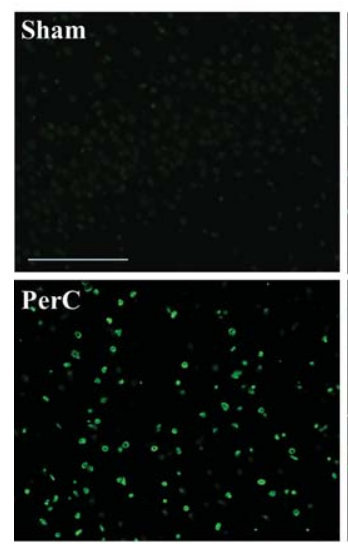

B

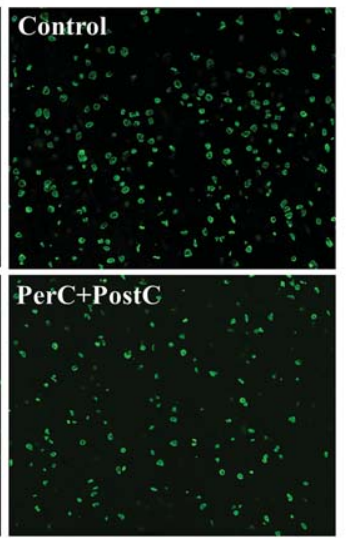

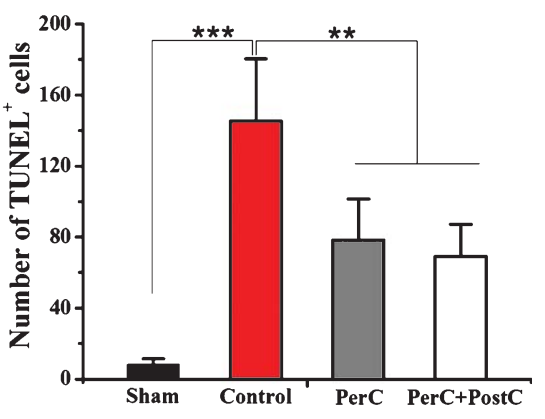

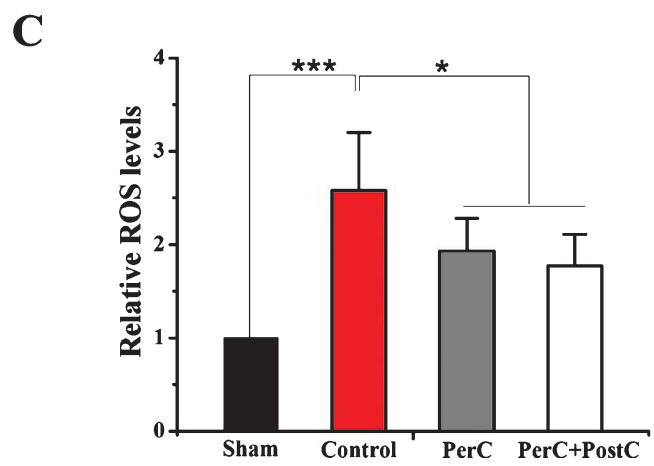

Fig. 2. Effect of per-conditioning and post-conditioning on DNA fragmentation and intracellular ROS levels. A, Representative TUNEL staining at 3 days after reperfusion. Scale bar $=100 \mu \mathrm{m}$. B, Quantification of average TUNEL positive cells number. ${ }^{* *} P<0.01$, ${ }^{* * *} P<0.001$. Error bars indicate SD. $N=5$ per group. C, Relative ROS levels in each group. ${ }^{*} P<0.05$. ${ }^{* * *} P<0.001$. Error bars indicate $\mathrm{SD}$. $N=4$ per group.

early episode of remote PerC reduces infarct size after myocardial infarction, and repeated remote PostC further reduces adverse left ventricular remodeling and improves survival in a dose-dependent fashion (Wei, et al., 2011). Consistent with these findings, our data show that PerC combined with daily PostC for up to 14 days significantly reduced the brain injury volume and ameliorated neurological deficits after focal ischemia. However, a single episode of PerC influence acute histological outcome after MCAO. Our results suggested that the therapeutic strategy of ischemic PerC in combination with PostC is necessary for long-term neuroprotection after MCAO. In contrast, a very recent study showed that not only remote PerC and PostC alone failed to reduce infarct size, but the combination of remote PerC+PostC also failed to reduce infarct size in a rat model of coronary artery occlusion as compared to the control group (Sachdeva, et al., 2014). Although the reason for this discrepancy remains unclear, the difference in animal species, the degree of ischemic stimulus, as well as the ischemic model employed may explain these contradicting results.

Yet, how the protective effect transmitted and communicated from the distant ischemic-reperfusion muscle to the brain remains a conundrum. A better understanding of the cellular and molecular mechanisms underlying remote ischemic conditioning may allow us to exploit the neuroprotective effect pharmaceutically. Up to date now, three potential mechanisms have been proposed: (1) Humoral factors released in the pre-conditioned organ are transported via the blood circulation to protect the target organ (Kanoria, et al., 2007), (2) Neurogenic transmission with involvement of muscle afferents and the autonomic nervous system (Kanoria, et al., 2007), and (3) Immunomodulation (Zhao, 2011). However, these proposed theories remain 

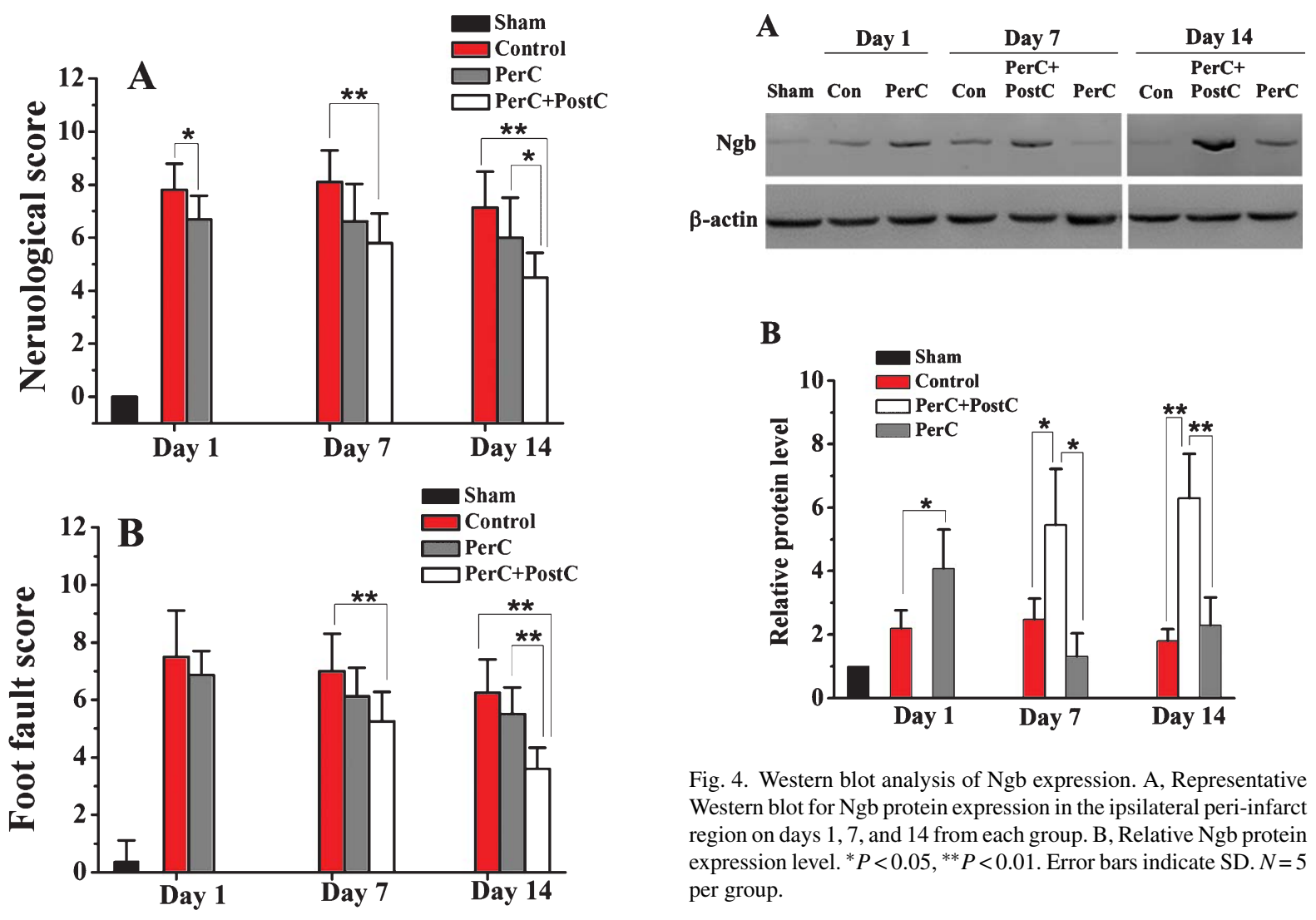

B

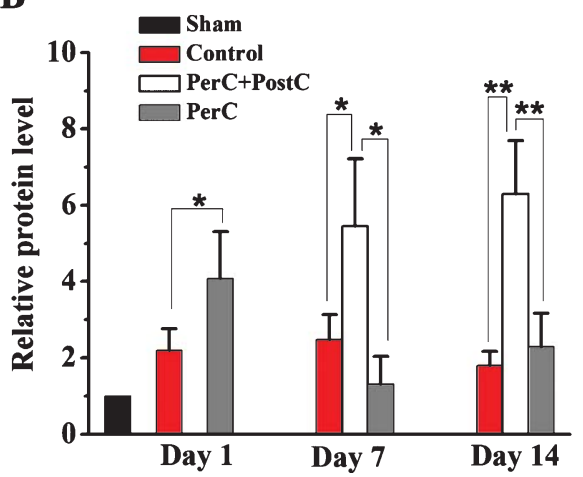

Fig. 4. Western blot analysis of Ngb expression. A, Representative Western blot for $\mathrm{Ngb}$ protein expression in the ipsilateral peri-infarct region on days 1,7 , and 14 from each group. B, Relative Ngb protein expression level. ${ }^{*} P<0.05,{ }^{* *} P<0.01$. Error bars indicate SD. $N=5$ per group.

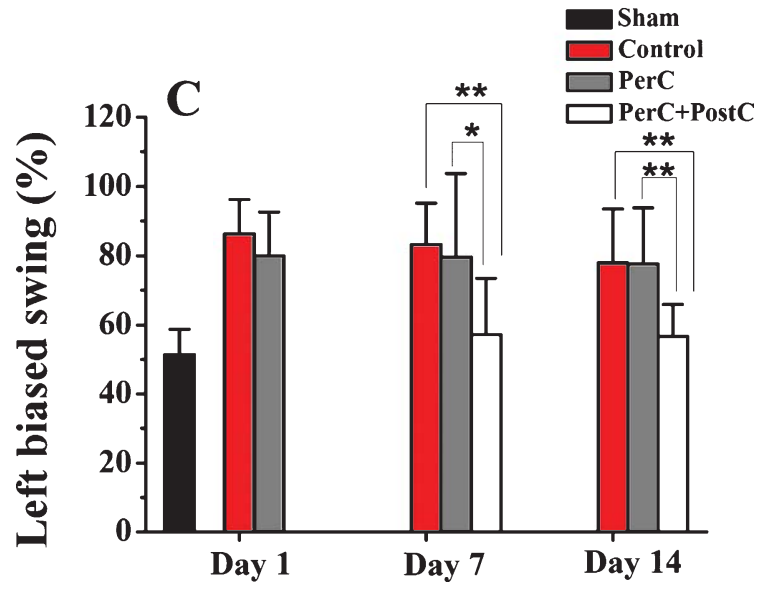

Fig. 3. Effect of per-conditioning and post-conditioning on neurobehavioral function after focal ischemia. A, Neurological deficits were determined using the neurobehavioral scoring system (higher scores correspond to more severe deficits). B, Motor function was determined by the ladder rung walking test (higher scores correspond to more severe deficits). C, Elevated Body Swing Test (higher percentage correspond to more severe deficits). ${ }^{*} P<0.05,{ }^{* *} P<0.01$. Error bars indicate SD. $N=10$ per group. to be clarified. In addition, several studies indicate that the underlying protective mechanisms of remote ischemic conditioning are associated with its ability to attenuate production of free radicals, promote the cell survival pathway, modulate the immune system, or to inhibit the apoptotic cell signaling pathways (Hoda, et al., 2012; Wang, et al., 2008; Xing, et al., 2008; Zhao, 2009; Zhao, et al., 2006). Ischemic preconditioning and post-conditioning prevent HIF-1 $\alpha$ mRNA induction in the rat liver after ischemia and reperfusion (Knudsen, et al., 2011). HIF-1 is essential for ischemia-induced angiogenesis, and may also be a critical mediator of late-phase pre-conditioning (Knudsen, et al., 2011). For these reasons, HIF-1 and the genes under its control such as Ngb may represent novel therapeutic targets for ameliorating the effects of ischemia in a variety of clinical settings. In parallel, Ngb has neuroprotective effects against stroke and neurodegenerative disorders (Burmester, et al., 2000; Yu, et al., 2012). Ngb expression is increased by neuronal hypoxia in vitro and focal cerebral ischemia in vivo (Shang, et al., 2006; Sun, et al., 2001), and Ngb overexpression protects 

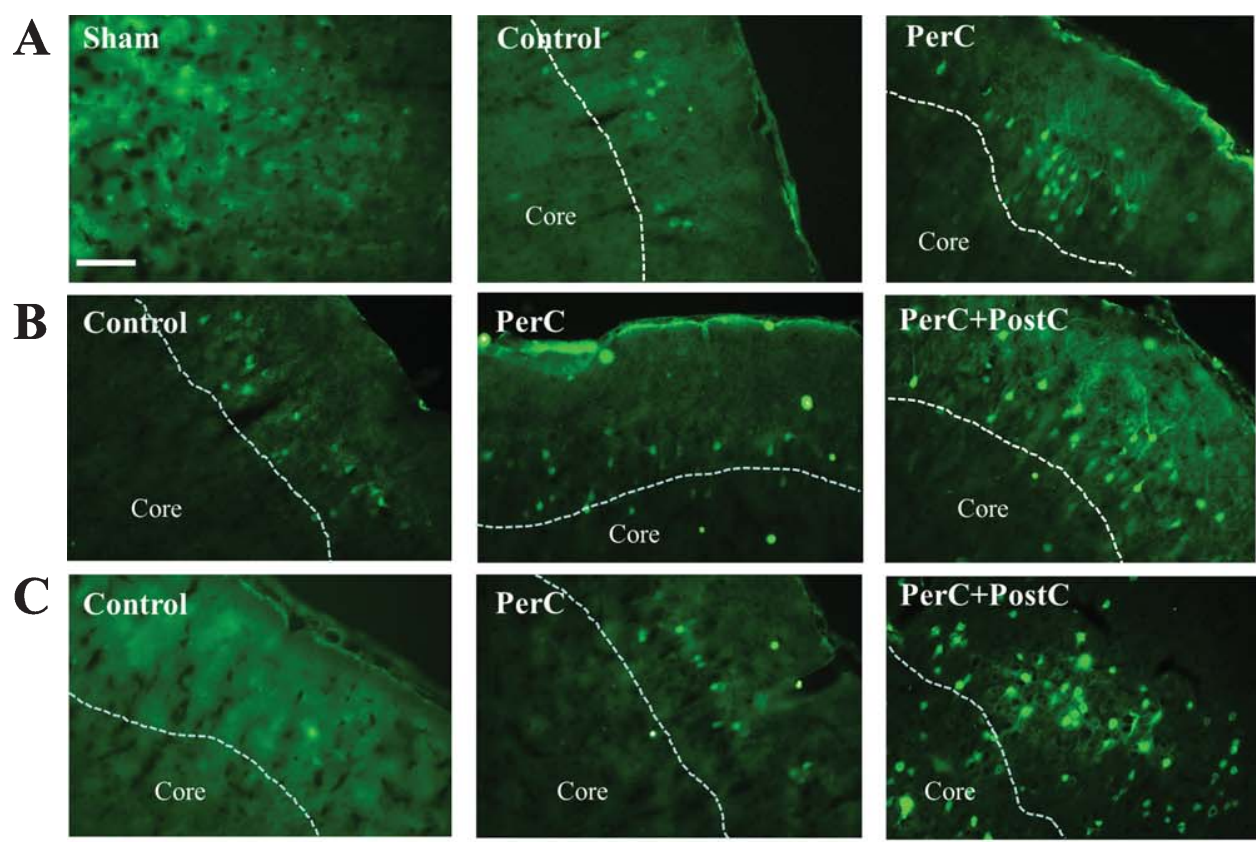

D
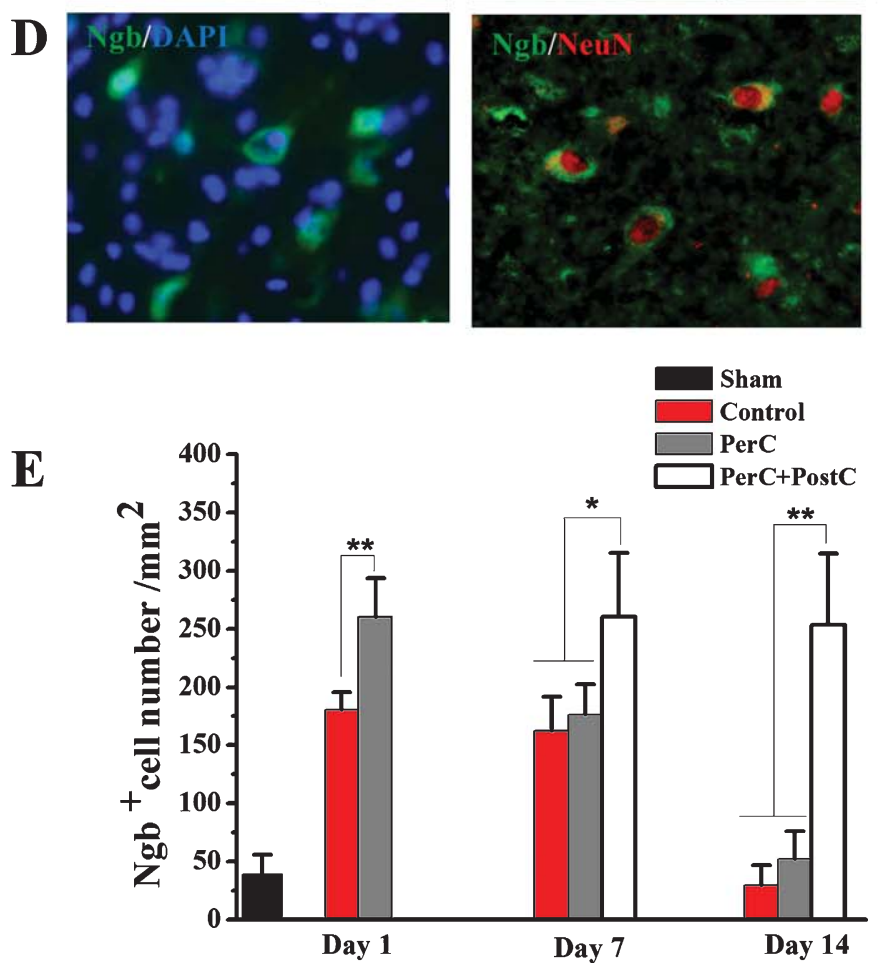

Fig. 5. Ngb expression pattern determined by immohistochemistry. A, Representative images of Ngb-positive cells in the peri-infarct region of each group at 1 day after reperfusion. B, Representative images of Ngb-positive cells in the peri-infarct region of each group at 7 days after reperfusion. C, Representative images of Ngb positive cells in the peri-infarct region of each group at 14 days after reperfusion. D, left, Representative images of Ngb- and DAPI-positive cells. Right, Double-label immunohistochemistry with antibodies against $\mathrm{Ngb}$ and neuron marker NeurN. Scale bar $=100 \mu \mathrm{m}$. E, Bar graphs depicting the number of Ngb-positive cells. Error bars indicate SD. ${ }^{*} P<0.05,{ }^{*} P<0.01 . N=5$ per group. Dashed line indicates the distinction between the ischemic core and peri-infarct region. 
neuronal cells from hypoxia/ischemia, ROS/NOS, or $\beta$-amyloid peptides (Jin, et al., 2008; Liu, et al., 2009; Ren, et al., 2013). Consistently, our data show that $\mathrm{Ngb}$ is significantly increased at 7 and 14 days after combined remote ischemic conditioning, compared with ischemic control group suggesting that $\mathrm{Ngb}$ may be involved in PerC+PostC-mediated neuroprotection after focal ischemia. However, direct causal relationship between Ngb expression and the neuroprotection of limb remote ischemia conditioning needs to be further confirmed.

In conclusion, our results suggest that the conventional PerC combined with PostC may be used as a long-term neuroprotective strategy against ischemiareperfusion injury and that $\mathrm{Ngb}$ may be one of the many important players in limb remote ischemia-mediated neuroprotection. These data may have clinical implications for the treatment of patients with cerebral ischemic stroke.

\section{Acknowledgments}

This work was partially supported by the National Basic Research Program of China (973 Program (No. 2011CB707804), National Natural Science Foundation of China (No. 81100979) and Scientific Special Funding of Capital Health Development (No. 20111001-03).

\section{Disclosures/Conflict of interest}

The authors have no conflicts of interest to declare.

\section{References}

Belayev, L., Alonso, O.F., Busto, R., Zhao, W., \& Ginsberg, M.D. (1996). Middle cerebral artery occlusion in the rat by intraluminal suture. Neurological and pathological evaluation of an improved model. Stroke 27(9), 1616-1622; discussion 23.

Borlongan, C.V., \& Sanberg, P.R. (1995). Elevated body swing test: A new behavioral parameter for rats with 6-hydroxydopamineinduced hemiparkinsonism. The Journal of Neuroscience: The Official Journal of the Society for Neuroscience, 15(7 Pt 2), 5372-5378.

Botker, H.E., Kharbanda, R., Schmidt, M.R., Bottcher, M., Kaltoft, A.K., Terkelsen, C.J., Munk, K., Andersen, N.H., Hansen, T.M., Trautner, S., Lassen, J.F., Christiansen, E.H., Krusell, L.R., Kristensen, S.D., Thuesen, L., Nielsen, S.S., Rehling, M., Sorensen, H.T., Redington, A.N., \& Nielsen, T.T. (2010). Remote ischaemic conditioning before hospital admission, as a complement to angioplasty, and effect on myocardial salvage in patients with acute myocardial infarction: A randomised trial. Lancet, 375(9716), 727-734.

Burmester, T., Weich, B., Reinhardt, S., \& Hankeln, T. (2000). A vertebrate globin expressed in the brain. Nature, 407(6803), 520-523.

Gidday, J.M. (2006). Cerebral preconditioning and ischaemic tolerance. Nat Rev Neurosci, 7(6), 437-448.

Haines, B., Demaria, M., Mao, X., Xie, L., Campisi, J., Jin, K., \& Greenberg, D.A. (2012). Hypoxia-inducible factor-1 and neuroglobin expression. Neuroscience Letters, 514(2), 137-140.

Hausenloy, D.J., \& Yellon, D.M. (2011). The therapeutic potential of ischemic conditioning: An update. Nat Rev Cardiol, 8(11), 619-629.

Hoda, M.N., Bhatia, K., Hafez, S.S., Johnson, M.H., Siddiqui, S., Ergul, A., Zaidi, S.K., Fagan, S.C., \& Hess, D.C. (2014). Remote ischemic perconditioning is effective after embolic stroke in ovariectomized female mice. Translational Stroke Research, 5(4), 484-490.

Hoda, M.N., Siddiqui, S., Herberg, S., Periyasamy-Thandavan, S., Bhatia, K., Hafez, S.S., Johnson, M.H., Hill, W.D., Ergul, A., Fagan, S.C., \& Hess, D.C. (2012). Remote ischemic perconditioning is effective alone and in combination with intravenous tissue-type plasminogen activator in murine model of embolic stroke. Stroke, 43(10), 2794-2799.

Hua, S., Antao, S.T., Corbett, A., \& Witting, P.K. (2010). The significance of neuroglobin in the brain. Curr Med Chem, 17(2), 160-172.

Jin, K., Mao, X., Xie, L., \& Greenberg, D.A. (2012). Interactions between vascular endothelial growth factor and neuroglobin. Neuroscience Letters, 519(1), 47-50.

Jin, K., Mao, X.O., Xie, L., Khan, A.A., \& Greenberg, D.A. (2008). Neuroglobin protects against nitric oxide toxicity. Neurosci Lett, 430(2), 135-137.

Jin, K., Mao, Y., Mao, X., Xie, L., \& Greenberg, D.A. (2010). Neuroglobin expression in ischemic stroke. Stroke; A journal of Cerebral Circulation, 41(3), 557-559.

Kanoria, S., Jalan, R., Seifalian, A.M., Williams, R., \& Davidson, B.R. (2007). Protocols and mechanisms for remote ischemic preconditioning: A novel method for reducing ischemia reperfusion injury. Transplantation, 84(4), 445-458.

Khan, A.A., Wang, Y., Sun, Y., Mao, X.O., Xie, L., Miles, E., Graboski, J., Chen, S., Ellerby, L.M., Jin, K., \& Greenberg, D.A. (2006). Neuroglobin-overexpressing transgenic mice are resistant to cerebral and myocardial ischemia. Proc Natl Acad Sci U S A, 103(47), 17944-17948.

Knudsen, A.R., Kannerup, A.S., Gronbaek, H., Andersen, K.J., Funch-Jensen, P., Frystyk, J., Flyvbjerg, A., \& Mortensen, F.V. (2011). Effects of ischemic pre- and postconditioning on HIF-1alpha, VEGF and TGF-beta expression after warm ischemia and reperfusion in the rat liver. Comparative Hepatology 10(1), 3.

Li, R.C., Guo, S.Z., Lee, S.K., \& Gozal, D. (2010). Neuroglobin protects neurons against oxidative stress in global ischemia. $J$ Cereb Blood Flow Metab, 30(11), 1874-1882.

Liu, J., Yu, Z., Guo, S., Lee, S.R., Xing, C., Zhang, C., Gao, Y., Nicholls, D.G., Lo, E.H., \& Wang, X. (2009). Effects 
of neuroglobin overexpression on mitochondrial function and oxidative stress following hypoxia/reoxygenation in cultured neurons. J Neurosci Res, 87(1), 164-170.

Meng, R., Asmaro, K., Meng, L., Liu, Y., Ma, C., Xi, C., Li, G., Ren, C., Luo, Y., Ling, F., Jia, J., Hua, Y., Wang, X., Ding, Y., Lo, E.H., \& Ji, X. (2012). Upper limb ischemic preconditioning prevents recurrent stroke in intracranial arterial stenosis. Neurology, 79(18), 1853-1861.

Moskowitz, M.A., Lo, E.H., \& Iadecola, C. (2010). The science of stroke: Mechanisms in search of treatments. Neuron, 67(2), 181-198.

Ren, C., Gao, M., Dornbos, D., 3rd, Ding, Y., Zeng, X., Luo, Y., \& Ji, $X$. (2012). Remote ischemic post-conditioning reduced brain damage in experimental ischemia/reperfusion injury. Neurol Res, 33(5), 514-519.

Ren, C., Li, Y., Han, R., Gao, D., Li, W., Shi, J., Hoogewijs, D., Braeckman, B.P., De Henau, S., Lu, Y., Qu, W., Gao, Y., Wu, Y., Li, Z., Liu, H., Wang, Z., \& Zhang, C. (2013). GLB-13 is associated with oxidative stress resistance in Caenorhabditis elegans. IUBMB Life, 65(5), 423-434.

Sachdeva, J., Dai, W., Gerczuk, P.Z., \& Kloner, R.A. (2014). Combined remote perconditioning and postconditioning failed to attenuate infarct size and contractile dysfunction in a rat model of coronary artery occlusion. J Cardiovasc Pharmacol Ther.

Shang, A., Zhou, D., Wang, L., Gao, Y., Fan, M., Wang, X., Zhou, R., \& Zhang, C. (2006). Increased neuroglobin levels in the cerebral cortex and serum after ischemia-reperfusion insults. Brain Res, 1078(1), 219-226.

Sun, F., Wang, X., Mao, X., Xie, L., Jin, K. (2012a). Ablation of neurogenesis attenuates recovery of motor function after focal cerebral ischemia in middle-aged mice. PloS one 7(10), e46326.

Sun, F., Xie, L., Mao, X., Hill, J., Greenberg, D.A., \& Jin, K. (2012b). Effect of a contralateral lesion on neurological recovery from stroke in rats. Restorative Neurology and Neuroscience, 30(6), 491-495.

Sun, Y., Jin, K., Mao, X.O., Zhu, Y., \& Greenberg, D.A. (2001). Neuroglobin is up-regulated by and protects neurons from hypoxic-ischemic injury. Proceedings of the National Academy of Sciences of the United States of America, 98(26), 1530615311.

Sun, Y., Jin, K., Peel, A., Mao, X.O., Xie, L., \& Greenberg, D.A. (2003). Neuroglobin protects the brain from experimental stroke in vivo. Proc Natl Acad Sci U S A, 100(6), 3497-3500.
Veighey, K., \& Macallister, R.J. (2012). Clinical applications of remote ischemic preconditioning. Cardiol Res Pract (2012), 620681.

Wang, J.Y., Shen, J., Gao, Q., Ye, Z.G., Yang, S.Y., Liang, H.W., Bruce, I.C., Luo, B.Y., \& Xia, Q. (2008). Ischemic postconditioning protects against global cerebral ischemia/reperfusioninduced injury in rats. Stroke, 39(3), 983-990.

Wang, X., Liu, J., Zhu, H., Tejima, E., Tsuji, K., Murata, Y., Atochin, D.N., Huang, P.L., Zhang, C., \& Lo, E.H. (2008). Effects of neuroglobin overexpression on acute brain injury and long-term outcomes after focal cerebral ischemia. Stroke, 39(6), 1869. 1874.

Wei, M., Xin, P., Li, S., Tao, J., Li, Y., Li, J., Liu, M., Zhu, W., \& Redington, A.N. (2011). Repeated remote ischemic postconditioning protects against adverse left ventricular remodeling and improves survival in a rat model of myocardial infarction. Circ Res, 108(10), 1220-1225.

Xing, B., Chen, H., Zhang, M., Zhao, D., Jiang, R., Liu, X., \& Zhang, S. (2008). Ischemic postconditioning inhibits apoptosis after focal cerebral ischemia/reperfusion injury in the rat. Stroke, 39(8), 2362-2369.

Yu, Z., Liu, N., Liu, J., Yang, K., \& Wang, X. (2012). Neuroglobin, a Novel Target for Endogenous Neuroprotection against Stroke and Neurodegenerative Disorders. Int J Mol Sci, 13(6), 69957014.

Zhang, X., Jizhang, Y., Xu, X., Kwiecien, T.D., Li, N., Zhang, Y., Ji, X., Ren, C., \& Ding, Y. (2014). Protective effects of remote ischemic conditioning against ischemia/reperfusion-induced retinal injury in rats. Visual Neuroscience, 31(3), 245-252.

Zhao, H. (2009). Ischemic postconditioning as a novel avenue to protect against brain injury after stroke. J Cereb Blood Flow Metab, 29(5), 873-885.

Zhao, H. (2011). The Protective Effects of Ischemic Postconditioning against Stroke: From Rapid to Delayed and Remote Postconditioning. The Open Drug Discovery Journal 5, 138-147.

Zhao, H., Sapolsky, R.M., \& Steinberg, G.K. (2006). Interrupting reperfusion as a stroke therapy: Ischemic postconditioning reduces infarct size after focal ischemia in rats. $J$ Cereb Blood Flow Metab, 26(9), 1114-1121.

Zwagerman, N., Plumlee, C., Guthikonda, M., \& Ding, Y. (2010). Toll-like receptor-4 and cytokine cascade in stroke after exercise. Neurol Res, 32(2), 123-126. 